\title{
Diagonal approximation of the form factor of the unitary group
}

\author{
G Berkolaiko \\ Department of Mathematics, Texas A\&M University, College Station TX 77840-3368, \\ USA \\ E-mail: Gregory.Berkolaiko@math.tamu.edu
}

\begin{abstract}
The form factor of the unitary group $U(N)$ endowed with the Haar measure characterizes the correlations within the spectrum of a typical unitary matrix. It can be decomposed into a sum over pairs of "periodic orbits", where by periodic orbit we understand any sequence of matrix indices. From here the diagonal approximation can be defined in the usual fashion as a sum only over pairs of identical orbits. We prove that as we take the dimension $N$ to infinity, the diagonal approximation becomes "exact", that is converges to the full form factor.
\end{abstract}

PACS numbers: 0545M, 0210D, 0250G, 0510G, 0270H 


\section{Introduction}

One of the central questions in the quantum theory of classically chaotic systems is whether the fluctuations in the spectra follow the predictions of random matrix theory (RMT). The spectral form factor, which is the Fourier transform of the two-point correlation function, is often used to characterize these fluctuations.

The advantage of the form factor is that it has a convenient expansion in terms of pairs of periodic orbits of the classical system. This expansion is an application of trace formulae (see [1] for a review) and has been a starting point for many investigations. The progress of understanding the role of periodic orbits in the universal behavior of the form factor of different systems is marked by such milestones as the diagonal approximation [2], the first off-diagonal contribution [3, 4] and the most recent preprint [5] outlining the complete expansion. Quantum graphs were also used [6] as a mathematical toy model with a simple-to-understand periodic orbit structure and an exact trace formula.

In a recent paper, Degli Esposti and Knauf [7] proposed to use the ultimate mathematical toy model for the random matrix conjecture - the random matrix theory itself. The authors argued that if one can perform a step-by-step expansion of the RMT form factor in terms of families of periodic orbits, it would greatly facilitate analogous expansions for other systems.

Degli Esposti and Knauf [7] studied the diagonal approximation to the form factor of one the random matrix ensembles, the Circular Unitary Ensemble (CUE). Their results, however, depend on a combinatorial conjecture, which is still unproven. The purpose of this letter is to present an alternative proof for their main result; our proof is unconditional and is significantly simpler. Our proof is based on the observation that the diagonal approximation can be expressed as a trace of certain doubly stochastic matrix. The asymptotic properties of the diagonal approximation can then be related to the spectral gap of the doubly stochastic matrix, which was previously studied by the author in [12].

The structure of the letter is as follows. In Section 2 we introduce the Circular Unitary Ensemble and the form factor. We follow with reviewing the main result of [7] and stating our main result in Section 3. The relevant properties of doubly stochastic matrices are reviewed in Section 4. Here we also prove our main result which follows quite simply from a technical lemma on the asymptotics of the second largest eigenvalue. This lemma is proved in Section 5 and we conclude with comparing our results with those of [7] in Section 6.

\section{Circular Unitary Ensemble and its form factor}

The Circular Unitary Ensemble is the unitary group $\mathrm{U}(N)$ equipped with the Haar measure:

Definition 1. $\mathrm{CUE}(N)$ is defined as the ensemble of all unitary $N \times N$ matrices endowed 
with the probability measure that is invariant under every automorphism

$$
\mathrm{U} \mapsto \mathrm{VUW},
$$

where $\mathbf{V}$ and $\mathbf{W}$ are any two $N \times N$ unitary matrices.

The form factor is a function describing the statistical properties of the spectrum of U. It is defined as the Fourier transform of the two-point spectral correlation function, $R_{2}(x)$

$$
R_{2, N}(x)=\left\langle\frac{1}{N} \sum_{j, k=1}^{N} \delta\left(x+\left(\phi_{j}-\phi_{k}\right) N\right)\right\rangle_{N},
$$

where $\exp \left(2 \pi i \phi_{j}\right)$ are the eigenvalues of $\mathbf{U}$ and the averaging is performed over the $\operatorname{CUE}(N)$. The form-factor of the unitary ensemble is thus given by

$$
K_{N}(t)=\frac{1}{N}\left\langle\left|\operatorname{Tr}\left(\mathbf{U}^{t}\right)\right|^{2}\right\rangle_{N} \quad(t \in \mathbb{Z}),
$$

The well-known formula for $K_{N}(t)$ is

$$
K_{N}(t)=\left\{\begin{array}{lc}
N, & t=0 \\
|t| / N, & 0<|t| \leq N \\
1, & N<|t|
\end{array} \quad(t \in \mathbb{Z}) .\right.
$$

This formula can, in particular, be derived by expanding the trace in (3),

$$
K_{N}=\frac{1}{N} \sum_{i_{1}, \ldots, i_{t}=1}^{N} \sum_{j_{1}, \ldots, j_{t}=1}^{N}\left\langle\prod_{k=1}^{t} U_{i_{k} i_{k+1}} U_{j_{k} j_{k+1}}\right\rangle_{N},
$$

where the convention $i_{t+1}=i_{1}$ is assumed here and throughout the manuscript. The above expression is analogous to the trace-formula expansions of the form factor in other systems, with $\mathbf{i}=\left(i_{1}, \ldots, i_{t}\right)$ and $\mathbf{j}=\left(j_{1}, \ldots, j_{t}\right)$ playing the role of the periodic orbits. It can be shown (see equation (8) below) that only the terms with $\mathbf{j}$ being a permutation of $\mathbf{i}$ contribute to the form factor, i.e.

$$
K_{N}=\frac{1}{N} \sum_{i_{1}, \ldots, i_{t}=1}^{N} \sum_{\sigma \in S_{t}}\left\langle\prod_{k=1}^{t} U_{i_{k} i_{k+1}} U_{i_{\sigma(k)} j_{\sigma(k+1)}}\right\rangle_{N},
$$

where $S_{t}$ is the group of all $t$-permutations (the symmetric group). This step is analogous to only considering pairs of orbits with a small action difference. The approximation to (6) that restricts the double sum even further, to pairs of periodic orbits that are equal (up to a cyclic shift), is called the diagonal approximation

$$
K_{1, N}=\frac{t}{N} \sum_{i_{1}, \ldots, i_{t}=1}^{N}\left\langle\prod_{k=1}^{t}\left|U_{i_{k} i_{k+1}}\right|^{2}\right\rangle_{N},
$$

where the factor $t$ comes from the number of possible shifts of an orbit. In this letter we compare the form factor $K_{N}(t)$ with its diagonal approximation.

The present article was inspired by a study by Degli Esposti and Knauf, [7. They proved that, assuming validity of a certain combinatorial conjecture, the diagonal 
approximation $K_{1, N}$ converges to $t / N$ uniformly in $t / N$ as $N \rightarrow \infty$. The convergence is proved in a closed interval which is bounded away from zero. The combinatorial conjecture requires a more thorough introduction and will be delayed to a later section.

Here we propose an alternative approach which exploits the fact that the sum in $K_{1, N}$ is the trace of a doubly stochastic matrix. Our proof is unconditional, i.e. it does not rely on the conjecture, and the interval of convergence is extended.

\section{Averages over unitary group and the main result}

The averages of products of the elements of matrices $\mathbf{U}$ have been studied by various authors, 8, 9, 10, 11]. Their main result is

$$
\left\langle U_{a_{1} b_{1}} \ldots U_{a_{s} b_{s}} U_{\alpha_{1} \beta_{1}}^{*} \ldots U_{\alpha_{t} \beta_{t}}^{*}\right\rangle_{N}=\delta_{t}^{s} \sum_{\sigma, \pi \in S_{t}} V_{N}\left(\sigma^{-1} \pi\right) \prod_{k=1}^{t} \delta_{a_{k}}^{\alpha_{\sigma(k)}} \delta_{b_{k}}^{\beta_{\pi(k)}},
$$

where $S_{t}$ is the symmetric group of permutations of the set $\{1, \ldots, t\}, \delta_{k}^{n}$ is the Kronecker delta and the coefficient $V_{N}\left(\sigma^{-1} \pi\right)$ depends only on the lengths of cycles in the cycle expansion of $\sigma^{-1} \pi$.

To introduce the results of [7] we need the following definitions.

Definition 2. Let $\sigma \in S_{t}$ and $i \in\{1,2, \ldots, t\}$. Then a cycle of $i$ with respect to $\sigma$ is the (finite) set $\left\{\sigma^{k}(i): k \in \mathbb{N}\right\}$. The cycle length of $i$ is the number of elements in the cycle of $i$. The rank $|\sigma|$ of a permutation $\sigma$ is the number of disjoint cycles induced by $\sigma$. By $|\sigma \vee \rho|$ we denote the number of disjoint sets of the form $\left\{\rho^{n} \sigma^{k}(i): k, n \in \mathbb{N}\right\}$. By $\tau$ we will denote the circular permutation $(1,2, \ldots, t)$. If $\sigma \in S_{t}, \sigma_{+}$will denote $\tau^{-1} \sigma \tau$.

Conjecture 1 (Degli Esposti - Knauf [7]). There exists a constant $C_{1}$ such that for all $t \leq N \in \mathbb{N}$

$$
\sum_{\pi \in S_{t}} V_{N}\left(\pi^{-1} \sigma\right) N^{\left|\pi \vee \sigma_{+}\right|} \leq C_{1} N^{\left|\sigma \vee \sigma_{+}\right|-t} \quad \sigma \in S_{t},
$$

Theorem 1 (Degli Esposti - Knauf [7]). Assuming Conjecture 1 is true, the form factor $K_{N}$ is approximated by the diagonal contribution in the following sense:

For all $\epsilon>0$

$$
\left|K_{N}(t)-K_{1, N}(t)\right| \rightarrow 0 \quad \text { as } N \rightarrow \infty
$$

uniformly in $\tau=t / N \in\left[\epsilon,(1-\epsilon) e / C_{1}\right]$.

Our main result is a version of the above theorem that drops the dependence on the Conjecture and extends the interval of convergence.

Theorem 2. For all $\epsilon>0$

$$
\left|t / N-K_{1, N}(t)\right| \rightarrow 0 \quad \text { as } N \rightarrow \infty
$$

uniformly in $\tau=t / N \in\left[\epsilon, \epsilon^{-1}\right]$. Thus, uniformly in $\tau=t / N \in[\epsilon, 1]$,

$$
\left|K_{N}(t)-K_{1, N}(t)\right| \rightarrow 0 \quad \text { as } N \rightarrow \infty .
$$




\section{Doubly stochastic matrices and the proof of Theorem 2}

Definition 3. An entry-wise nonnegative $N \times N$ matrix $\mathbf{M}$ is called doubly stochastic if

$$
\sum_{i=1}^{N} M_{i, j}=1 \quad \forall j \quad \text { and } \quad \sum_{j=1}^{N} M_{i, j}=1 \quad \forall i .
$$

The set of all such $N \times N$ matrices we denote by $\operatorname{DS}(N)$. $\operatorname{DS}(N)$ is a monoid with respect to the matrix multiplication, in particular if $\mathbf{A}$ and $\mathbf{B}$ are doubly stochastic then so is $\mathbf{A B}$.

It follows directly from the definition that the vector $\mathbf{e}=(1 / N, \ldots, 1 / N)$ is both a left and a right eigenvector of any matrix from $\operatorname{DS}(N)$. The corresponding eigenvalue 1 is the largest (by modulus) one. The following observation shows the importance of the asymptotics of the second largest eigenvalue.

If $\mathbf{U}$ is unitary then the matrix $\mathbf{M}$ defined by

$$
M_{i, j}=\left|U_{i, j}\right|^{2} .
$$

is doubly stochastic. It is then easy to see that

$$
\operatorname{Tr} \mathbf{M}^{t}=\sum_{j=1}^{N}\left(\mathbf{M}^{t}\right)_{j, j}=\sum_{i_{1}, \ldots, i_{t}=1}^{N} \prod_{k=1}^{t}\left|U_{i_{k} i_{k+1}}\right|^{2},
$$

and, therefore, the diagonal approximation $K_{1, N}$ is equal to

$$
K_{1, N}=\frac{t}{N}\left\langle\operatorname{Tr} \mathbf{M}^{t}\right\rangle_{N}
$$

where the averaging is performed with respect to the measure induced on doubly stochastic matrices by the correspondence (14). In [12] it was speculated that the second largest eigenvalue of such random matricesł $\mathbf{M}$ decay, on average, as $N^{-1 / 2}$. If this is indeed so, we immediately get

$$
\left\langle\operatorname{Tr} \mathbf{M}^{t}\right\rangle_{N}=1+\sum_{i=2}^{N}\left\langle\lambda_{i}^{t}\right\rangle \approx 1+(N-1) N^{-t / 2} \rightarrow 1,
$$

for $t>2$, which would prove Theorem 2. While the conjecture of [12] is still unproven, we can use the technique of [12] to achieve the same result without directly calculating the eigenvalues of $\mathbf{M}$.

Since the eigenvalues of $\mathbf{M}$ lie in the unit circle, it is intuitively clear that $\operatorname{Tr} \mathbf{M}^{t}=\sum \lambda_{i}^{t}$ should be a decreasing function in $t$, at least "on average". It is therefore tempting to show that $\left\langle\operatorname{Tr} \mathbf{M}^{t}\right\rangle_{N} \rightarrow 1$ for some fixed $t$ as $N \rightarrow \infty$ and then conclude that this property still holds if we send $t$ to infinity as well. Indeed, this would work if the eigenvalues of $\mathbf{M}$ were positive real numbers, which is generically not the case for matrices $\mathbf{M}$. To circumvent this difficulty, we form a matrix

$$
\mathbf{A}=\mathbf{M}^{T} \mathbf{M}
$$

$\ddagger$ an extensive discussion of properties of such random matrices is contained in 13 
which is symmetric, doubly stochastic and positive definite. Now we can estimate the eigenvalues of $\mathbf{M}$ through the eigenvalues of $\mathbf{A}$.

Proposition 1 ([12]). Second largest eigenvalues of $\mathbf{M}$ and $\mathbf{A}$ satisfy

$$
\left|\lambda_{2}(\mathbf{M})\right|^{2} \leq \lambda_{2}(\mathbf{A}) \text {. }
$$

Proof. Let $\mathbf{v}$ be the eigenvector of $\mathbf{M}$ corresponding to $\lambda_{2}(\mathbf{M})$ and orthogonal to e. Such eigenvector always exists: if $\lambda_{2}(\mathbf{M}) \neq 1$, then we write

$$
(\mathbf{v}, \mathbf{e})=\left(\mathbf{v}, \mathbf{M}^{T} \mathbf{e}\right)=(\mathbf{M} \mathbf{v}, \mathbf{e})=\lambda(\mathbf{v}, \mathbf{e}),
$$

and therefore $(\mathbf{v}, \mathbf{e})=0$. If $\lambda_{2}(\mathbf{M})=1$, the geometric multiplicity of 1 is equal to its algebraic multiplicity (see, e.g. [14]) and we can find $\mathbf{v}$ by orthogonalization.

Then, since all eigenvectors of $A$ are orthogonal, we have

$$
\lambda_{2}(\mathbf{A})=\max _{|\mathbf{u}|=1,(\mathbf{u}, \mathbf{e})=0}(\mathbf{A u}, \mathbf{u}) \geq(\mathbf{A v}, \mathbf{v})=(\mathbf{M v}, \mathbf{M v})=\left|\lambda_{2}(\mathbf{M})\right|^{2},
$$

Now the idea is to find the power $k$ such that $\left\langle\lambda(\mathbf{A})^{k}\right\rangle_{N}$ decay sufficiently fast. It turns out that $k=3$ is enough.

Lemma 1. The Haar probability measure on the unitary group induces a measure on symmetric doubly stochastic matrices $\mathbf{A}$ via (14) and (18). With respect to this measure $\left\langle\lambda_{2}(\mathbf{A})^{3}\right\rangle_{N}=O\left(N^{-2}\right)$.

The proof is rather technical and will be given in a later section. This lemma enables us to give a simple proof of our main result.

Proof of Theorem 2. We show that $\left\langle\operatorname{Tr} \mathbf{M}^{t}\right\rangle \rightarrow 1$. If $\tau \geq \epsilon$ and $N$ is sufficiently large then $t=\tau N \geq 6$, leading to

$$
\begin{aligned}
\left|\left\langle\operatorname{Tr} \mathbf{M}^{t}\right\rangle-1\right| & =\left|\left\langle\sum_{k=2}^{N} \lambda_{k}(\mathbf{M})^{t}\right\rangle\right| \leq \sum_{k=2}^{N}\left|\left\langle\lambda_{k}(\mathbf{M})^{t}\right\rangle\right| \leq \sum_{k=2}^{N}\left\langle\left|\lambda_{k}(\mathbf{M})\right|^{t}\right\rangle \\
& \leq(N-1)\left\langle\left|\lambda_{2}(\mathbf{M})\right|^{t}\right\rangle \leq(N-1)\left\langle\left|\lambda_{2}(\mathbf{M})\right|^{6}\right\rangle \\
& \leq(N-1)\left\langle\left|\lambda_{2}(\mathbf{A})\right|^{3}\right\rangle=O\left(N^{-1}\right) .
\end{aligned}
$$

Now we use equation (16) to obtain the sought after conclusion.

\section{Proof of Lemma 1}

We start with the trace of $\mathbf{A}^{3}$,

$$
\operatorname{Tr} \mathbf{A}^{3}=1+\sum_{k=2}^{N} \lambda_{k}(\mathbf{A})^{3} \geq 1+\lambda_{2}(\mathbf{A})^{3},
$$


since all eigenvalues of $\mathbf{A}$ are non-negative real numbers. Thus we can estimate $0 \leq \lambda_{2}(\mathbf{A})^{3} \leq \operatorname{Tr} \mathbf{A}^{3}-1$. Writing out the trace we get

$\operatorname{Tr} \mathbf{A}^{3}=\operatorname{Tr}\left(\mathbf{M}^{T} \mathbf{M} \mathbf{M}^{T} \mathbf{M} \mathbf{M}^{T} \mathbf{M}\right)$

$$
\begin{aligned}
& =\sum_{i, j, k, l, m, n=1}^{N}\left(\mathbf{M}^{T}\right)_{i, j}(\mathbf{M})_{j, k}\left(\mathbf{M}^{T}\right)_{k, l}(\mathbf{M})_{l, m}\left(\mathbf{M}^{T}\right)_{m, n}(\mathbf{M})_{n, i} \\
& =\sum_{i, j, k, l, m, n=1}^{N}\left|U_{j, i} U_{j, k} U_{l, k} U_{l, m} U_{n, m} U_{n, i}\right|^{2} .
\end{aligned}
$$

Now we average over the unitary group,

$$
\left\langle\lambda(\mathbf{A})^{3}\right\rangle_{N}+1 \leq \sum_{i, j, k, l, m, n=1}^{N}\left\langle\left|U_{j, i} U_{j, k} U_{l, k} U_{l, m} U_{n, m} U_{n, i}\right|^{2}\right\rangle_{N} .
$$

At this point we can apply equation (18) with $\alpha_{k}=a_{k}$ and $\beta_{k}=b_{k}$ with $1 \leq k \leq 6$. The indices are $\mathbf{a}=(j, j, l, l, n, n)$ and $\mathbf{b}=(i, k, k, m, m, i)$. We denote

$$
\left\langle\left|U_{j, i} U_{j, k} U_{l, k} U_{l, m} U_{n, m} U_{n, i}\right|^{2}\right\rangle=W_{i, k, m}^{j, l, n} .
$$

To cut down the number of the coefficients $W$ that need to be calculated, we list some of the properties arising from the symmetries of (8).

(i) Application of a permutation to both $\mathbf{a}$ and $\mathbf{b}$ leaves $W$ invariant. Due to the special form of $\mathbf{a}$ and $\mathbf{b}$, the only eligible permutations are shifts:

$$
W_{i, k, m}^{j, l, n}=W_{m, i, k}^{n, j, l}=W_{k, m, i}^{l, n, j}
$$

(ii) We can switch around $\mathbf{a}$ and $\mathbf{b}$. After that we should apply a right shift to bring them back to the special form: $\mathbf{a}^{\prime}=(i, i, k, k, m, m)$ and $\mathbf{b}^{\prime}=(n, j, j, l, l, n)$. Thus,

$$
W_{i, k, m}^{j, l, n}=W_{n, j, l}^{i, k, m} .
$$

Note that the lower indices on the right-hand side are shifted from their original order.

(iii) The coefficients $W$ do not depend on the individual values of $j, l$ and $n$, but only on whether they are pairwise distinct. That is, if

$$
j=l \Leftrightarrow j^{\prime}=l^{\prime}, \quad l=n \Leftrightarrow l^{\prime}=n^{\prime} \quad \text { and } \quad n=j \Leftrightarrow n^{\prime}=j^{\prime},
$$

then

$$
W_{i, k, m}^{j, l, n}=W_{i, k, m}^{j^{\prime}, l^{\prime}, n^{\prime}}
$$

The same applies to $i, k$ and $m$.

Using these properties we can list all possible values of $W$. If $\{j, l, n\}$ are pairwise distinct, $W_{i, k, m}^{j, l, n}=W_{i, k, m}^{1,2,3}$ and there are $N(N-1)(N-2)$ ways to select such $j, l, n$. If $l=n$ but $j$ is different, $W_{i, k, m}^{j, l, n}=W_{i, k, m}^{1,2,2}$ and there are $N(N-1)$ way to choose $j$ and $n$; the cases $j=l \neq n$ and $j=n \neq l$ are analogous. Finally, when $j=l=n$, $W_{i, k, m}^{j, l, n}=W_{i, k, m}^{1,1,1}$ and there are $N$ ways to choose the value of $j$. The dependence on indices $\{i, k, m\}$ can be treated in the same way. Table 1 lists all contributing terms 


\begin{tabular}{|c|c|c|c|}
\hline Contributing terms & Notation & Value & Number of occurrences \\
\hline$W_{1,2,3}^{1,2,3}$ & $w_{33}$ & $\frac{N^{5}+6 N^{4}+5 N^{3}-20 N^{2}-4 N+32}{D(N-2)\left(N^{2}-1\right)(N-1) N}$ & $N^{2}(N-1)^{2}(N-2)^{2}$ \\
\hline$W_{1,2,2}^{1,2,2}, W_{1,2,3}^{2,1,2}, W_{1,2,3}^{2,2,1}$ & $w_{23}$ & $\frac{2\left(N^{2}+5 N+8\right)}{D(N-1) N}$ & $N^{2}(N-1)^{2}(N-2)$ \\
\hline$W_{1,2,2}^{1,2}, W_{2,1,2}^{2,1,2}, W_{2,2,1}^{2,2,1}$ & $w_{22 a}$ & $\frac{6\left(N^{2}+3 N+6\right)}{D(N-1) N}$ & $N^{2}(N-1)^{2}$ \\
\hline$W_{2,1,2}^{1,2,2}, W_{2,2,1}^{2,1,2}, W_{1,2,2}^{2,2,1}$ & $w_{22 b}$ & $\frac{6\left(N^{2}+3 N+6\right)}{D(N-1) N}$ & $N^{2}(N-1)^{2}$ \\
\hline$W_{2,2,1}^{1,2,2}, W_{1,2,2}^{2,1,2}, W_{2,1,2}^{2,2,1}$ & $w_{22 c}$ & $\frac{8(N+2)(N+1)}{D(N-1) N}$ & $N^{2}(N-1)^{2}$ \\
\hline$W_{1,2,3}^{1,1,1}$ & $w_{13}$ & $\frac{8}{D}$ & $N^{2}(N-1)(N-2)$ \\
\hline$W_{1,2,2}^{1,1,1}, W_{2,1,2}^{1,1,1}, W_{2,2,1}^{1,1,1}$ & $w_{12}$ & $\frac{48}{D}$ & $N^{2}(N-1)$ \\
\hline$W_{1,1,1}^{1,1,1}$ & $w_{11}$ & $\frac{720}{D}$ & $N^{2}$ \\
\hline
\end{tabular}

Table 1. Factors contributing to $\left\langle\operatorname{Tr} \mathbf{A}^{3}\right\rangle$. We use notation $D=(N+5)(N+4)(N+$ $3)(N+2)(N+1) N$. The remaining terms can be obtained using $W_{i, k, m}^{j, l, n}=W_{n, j, l}^{i, k, m}$. Whenever the first column lists three terms, the last column gives the number of occurrences of each of those terms.

together with the number of times they occur in expansion (23). The values of $W$ were computed using a program which utilizes formula (8) and the data for $V_{N}$ from [8].

Thus we get

$$
\begin{aligned}
\left\langle\operatorname{Tr} \mathbf{A}^{3}\right\rangle_{N}= & N^{2}(N-1)^{2}(N-2)^{2} w_{33}+2 \times 3 N^{2}(N-1)^{2}(N-2) w_{23} \\
& +3 N^{2}(N-1)^{2} w_{22 a}+3 N^{2}(N-1)^{2} w_{22 b}+3 N^{2}(N-1)^{2} w_{22 c} \\
& +2 \times N^{2}(N-1)(N-2) w_{13}+2 \times 3 N^{2}(N-1) w_{12}+N^{2} w_{11},
\end{aligned}
$$

where the factors of 2 arise due to $W_{i, k, m}^{j, l, n}=W_{n, j, l}^{i, k, m}$. The result is

$$
\begin{aligned}
& \left\langle\operatorname{Tr} \mathbf{A}^{3}\right\rangle_{N}=\frac{N^{6}+16 N^{5}+105 N^{4}+370 N^{3}+716 N^{2}+348 N-136}{(N+1)^{2}(N+5)(N+4)(N+3)(N+2)} \\
& =1+5 N^{-2}+O\left(N^{-3}\right),
\end{aligned}
$$

which, together with inequality $\lambda_{2}(\mathbf{A})^{3} \leq \operatorname{Tr} \mathbf{A}^{3}-1$, finishes the proof.

\section{Discussion}

We presented an alternative and complete proof of the main result of [7]. However the main importance of the results of [7] lies in the method of their derivation and in establishing a foothold in understand the combinatorics of the form factor expansion. Below we formulate two conjectures which can be studied using the methods of [7].

The first conjecture is rephrasing an observation made in [12] regarding the asymptotics of the eigenvalues of doubly stochastic matrices $\mathbf{M}$.

Conjecture 2. For any $j \geq 2$ and $k\left\langle\left|\lambda_{j}(\mathbf{M})\right|^{k}\right\rangle=O\left(N^{-k / 2}\right)$, where the averaging is performed with respect to the measure induced by (14). 
The second conjecture is a strengthening of our result which would follow if Conjecture 2 were proved. It concerns the speed of convergence of the diagonal approximation

Conjecture 3. For any $\tau \in(0, \infty)$

$$
\left\langle\operatorname{Tr} \mathbf{A}^{\tau N}\right\rangle-1 \rightarrow 0
$$

faster than any power of $N$.

[1] Cohen D, Primack H and Smilansky U 1998 Ann. Physics 264(2) 108-170 (Preprint chao-dyn/9708017)

[2] Berry M V 1985 Proc. R. Soc. Lond. A 400 229-251

[3] Sieber M 2002 J. Phys. A 35(42) L613-L619

[4] Sieber M and Richter K 2001 Physica Scripta T90 128

[5] Muller S, Heusler S, Braun P, Haake F and Altland A 2005 Periodic-orbit theory of universality in quantum chaos (Preprint nlin.CD/0503052)

[6] Berkolaiko G, Schanz H and Whitney R S 2003 J. Phys. A 36(31) 8373-8392 (Preprint nlin.CD/0205014)

[7] Degli Esposti M and Knauf A 2004 J. Math. Phys. 45(12) 4956-4979 (Preprint cond-mat/0311074)

[8] Samuel S 1980 J. Math. Phys. 21(12) 2695-2703

[9] Ullah N 1964 Nucl. Phys. 58 65-71

[10] Mello P A 1990 J. Phys. A 23(18) 4061-4080

[11] Brouwer P W and Beenakker C W J 1996 J. Math. Phys. 37(10) 4904-4934 (Preprint cond-mat/9604059)

[12] Berkolaiko G 2001 J. Phys. A 34(22) L319-L326 (Preprint nlin.CD/0104009)

[13] Życzkowski K, Kuś M, Słomczyński W and Sommers H J 2003 J. Phys. A 36(12) 3425-3450 (Preprint nlin.CD/0112036)

[14] Gantmacher F R 1959 The theory of matrices (New York: Chelsea) 